\title{
Explicating the Applications of Quality by Design Tools in Optimization of Microparticles and Nanotechnology Based Drug Delivery Systems
}

\author{
Neelam Sharma ${ }^{1}(\mathbb{D})$, Sukhbir Singh $^{1}(\mathbb{D})$, Tapan Behl $^{1}{ }^{(\mathbb{D})}$, Nidhi Gupta $^{1}{ }^{(\mathbb{D})}$, Ritu Gulia $^{1,3}$, \\ Neha Kanojia $1,2, *$ iD

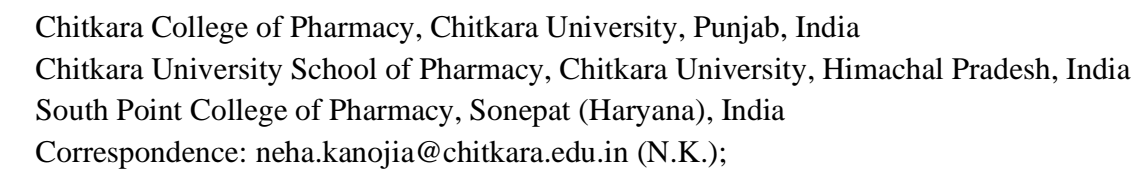

Scopus Author ID: 57216373425

Received: 25.06.2021; Revised: 26.07.2021; Accepted: 30.07.2021; Published: 15.08.2021

\begin{abstract}
Quality by design (QbD) can also contribute to design, manufacturing, and producing highly finished goods. To better explain the manufacturing processes, the FDA focused $\mathrm{QbD}$ in the healthcare industry, based on a comprehensive understanding of how technology and design parameters affect the quality of the manufactured product. Various elements of QbD are critical quality attributes (CQA); critical material attributes (CMAs), and critical process parameters (CPPs). The tools generally applied in $\mathrm{QbD}$ are risk assessment, design of experiments, and process analytical technology. The various benefits of the QbD model are preventing sampling errors and variability in research studies, less experimentation, and enhanced productivity. Since the microparticles and nanotechnology-based formulations need complex experimentation and an extremely time-consuming process, the application of $\mathrm{QbD}$ tools in such investigations can intelligently conclude the research processes. This review article provides a brief outline of the fundamentals, elements, and tools of QbD. Furthermore, the recently published applications of QbD in the optimization of microparticles and nanotechnology-based drug delivery systems have been discussed in this review.
\end{abstract}

Keywords: quality by design; critical quality attributes; critical material attributes; critical process parameters; experimentation design; process analytical technology; microparticles; nanotechnology.

(C) 2021 by the authors. This article is an open-access article distributed under the terms and conditions of the Creative Commons Attribution (CC BY) license (https://creativecommons.org/licenses/by/4.0/).

\section{Introduction}

The primary purpose of drug manufacturing is to provide a higher quality-based product and ensure that acceptable quality is reliably produced. Technological expertise is required to access data collected from experimental studies and manufacturing to acknowledge design, specifications, and system control authenticity. Alterations in the manufacturing process and preparation and development are seen as opportunities to gain new knowledge or stimulate system design development [1-5]. Quality by design (QbD) is the science of developing and building formulations and product lines that meet predestined criteria [6-8]. Like other regulatory guidelines, for example, International Council for Harmonization (ICH) Q8, Q9, and Q10, and current guidance documents, current good manufacturing procedures (cGMP), food and drug administration (FDA), currently, the principles of QbD have gained popularity for several interventions in drug discovery in the twenty-first century [9-16]. Numerous https://biointerfaceresearch.com/ 
benefits of the QbD model are avoiding sampling errors and variability in investigations, escaping from prolonged experimentations, preventing complications with governing compliance, and enhancing production alternatives [17-19]. Figure 1 depicts the various processes required in the QbD model [20,21].

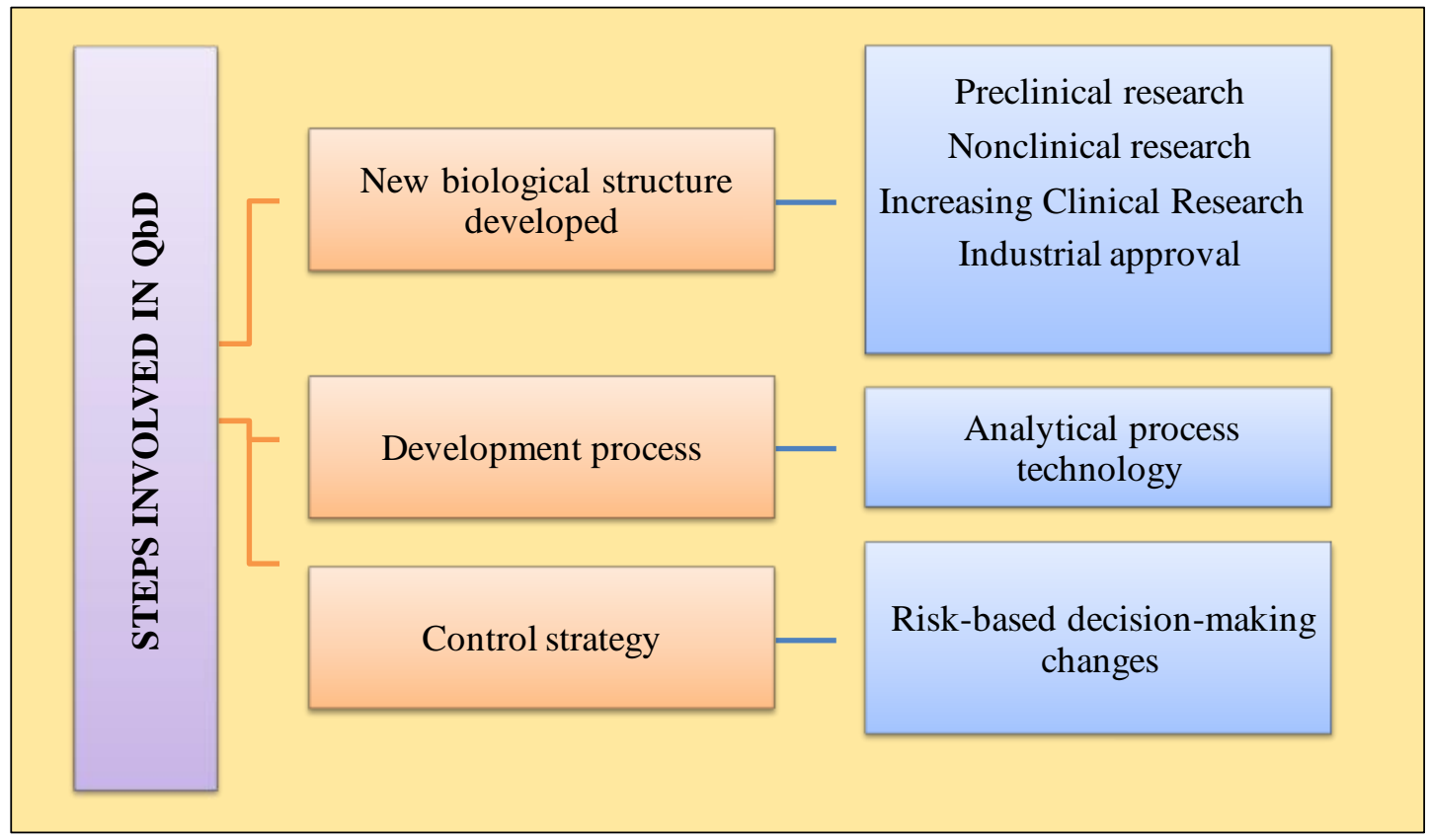

Figure 1. Pictorial representation of the progression of quality by design model.

The quality by test (QbT) approach maintains product quality through adopting a number of protocols, i.e., raw material testing, the manufacturing cycle of defined pharmaceutical drugs, and finished product testing in anticipation to fulfill FDA requirements and certain additional conditions to bring the products into the industry for bulk manufacturing consequently. Due to the lack of knowledge of the procedure and uncertainty over it, the primary causes of failure have yet to be determined. Therefore, suppliers could subsequently regenerate the cycle before the underlying causes of inaccuracy are identified and treated, and furthermore, reevaluate that the inclusion process has been followed to surpass through unsuccessful conditions [21]. Table 1 shows a comparison of QbT and QbD [22].

Table 1. Comparison analysis between $\mathrm{QbT}$ and $\mathrm{QbD}$.

\begin{tabular}{|c|c|c|c|}
\hline & & & \\
\hline Constraints & QbD & QbT & Interpretation \\
\hline $\begin{array}{l}\text { Product } \\
\text { development } \\
\text { process }\end{array}$ & $\begin{array}{l}\text { The criteria } \\
\text { concentrated on } \\
\text { interpreting } \\
\text { quality parameters }\end{array}$ & $\begin{array}{l}\text { A norm depending on a } \\
\text { batch's history }\end{array}$ & $\begin{array}{l}\text { The QbT strategy is based on accuracy and } \\
\text { also excludes or denied uncertainty. } \\
\text { Flexible technique within the layout which } \\
\text { enables long-term development in QbD } \\
\text { system. }\end{array}$ \\
\hline Validation & $\begin{array}{l}\text { The model must be } \\
\text { authenticated regularly }\end{array}$ & $\begin{array}{l}\text { The focus is on } \\
\text { reproducibility. Adaptable } \\
\text { in the design field }\end{array}$ & $\begin{array}{l}\text { In the QbT method, preliminary samples must } \\
\text { be validated. The QbD technique emphasizes } \\
\text { control policy. }\end{array}$ \\
\hline $\begin{array}{l}\text { Risk-based } \\
\text { management }\end{array}$ & $\begin{array}{l}\text { Power shifted } \\
\text { dramatically; real-time } \\
\text { issue }\end{array}$ & $\begin{array}{l}\text { Changes in regulatory } \\
\text { priority requirements } \\
\text { Prior authorization }\end{array}$ & $\begin{array}{l}\text { Value estimation is separated from research, } \\
\text { and risk assessment has been carried out using } \\
\text { the QbT method. In the QbD method, the } \\
\text { decision was based on process identification } \\
\text { and risk management. }\end{array}$ \\
\hline $\begin{array}{l}\text { Lifecycle } \\
\text { based } \\
\text { management }\end{array}$ & $\begin{array}{l}\text { Constant adjustment } \\
\text { within the process }\end{array}$ & $\begin{array}{l}\text { Reacting to issues; space } \\
\text { layout }\end{array}$ & $\begin{array}{l}\text { Approval modifications are required in the } \\
\text { QbT design, while change is made completely } \\
\text { throughout the QbD model. }\end{array}$ \\
\hline
\end{tabular}




\begin{tabular}{l|l|l|l}
\hline Constraints & QbD & QbT & Interpretation \\
\hline $\begin{array}{l}\text { Process } \\
\text { control }\end{array}$ & $\begin{array}{l}\text { Quality assurance is } \\
\text { maintained on an } \\
\text { ongoing basis }\end{array}$ & $\begin{array}{l}\text { Go/No-Go, Tracking, } \\
\text { Comprehensions, } \\
\text { Evaluation }\end{array}$ & $\begin{array}{l}\text { Surveillance and examination ensure } \\
\text { performance in the QbT strategy. By } \\
\text { definition, the QbD strategy is theoretically } \\
\text { oriented. }\end{array}$
\end{tabular}

\section{Elements of QbD}

In order to address GMP's limitations, the FDA introduced cGMP in the year 2002. According to cGMP, the focus on "software" throughout the production phase, especially at the managerial level, precisely defines the transparency of workers [23-26]. The ICH Q8 framework defines $\mathrm{QbD}$ as a systematic approach for development, which begins from predefined targets and emphasizes process and product evaluation with tracking, based on validated quality and process risk assessment [9]. The various research studies of drug development and industrial experience offer knowledge and experiences that help create quality standards and controls [27].

\subsection{Setting a goal.}

The quality goal profile (QTP) has been the center of QbD, which serves as the foundation for product design and development in respect to the initial target criteria stated within QbD definition. The quality target product profile (QTPP) establishes the quality of the finished product. In the case of analytic process development, this is frequently known as target product profile (TPP), as it includes an analytical target profile (ATP). TPP may play a significant part in all drug discovery procedures, including scheduling, choice, training, and execution of clinical research methods [28].

\subsection{Critical quality attributes (CQA) assessment.}

The CQAs are commonly related to the drug material, inert components, intermediate inputs (additives or excipients), and the dosage form itself. CQAs typically influence the characteristics of drug products, like particle sizes, drug release, solubility, zeta potential, entrapment efficiency, product yield, and drug loading [29]. The mechanical, physical, microbial, or biological characteristics or qualities of raw materials are described as critical material attributes (CMAs). CMAs are utilized during the context of a suitable range collection or production to assure that product content and excipients are consistent. This information serves as a foundation for applying the CQA to the product's effectiveness. The QbD paradigm is unique in that it uses rigorous risk assessment methods to identify CQAs. CQAs in large oral delivery forms typically affect drug release, quality, safety, and strength [9].

\subsection{Planning and implementing a control-based strategy.}

The goal of control-based planning is always to keep the product safe, and the system stays within the expected lowest and the highest limits. The factors and products are reviewed regularly during the production process, which ensures robustness. Typically, hit and trial frameworks are scaled up. Critical process parameters (CPPs) are parameters that influence CQAs and should therefore be monitored or controlled to ensure that the process produces the correct performance. Process robustness is defined as a system's ability to maintain rational effectiveness and efficiency while accepting input variance [30]. CQAs are concerned with 
production parts, while CMAs are concerned with raw resources, like drug products and inert materials used in the manufacturing process. In general, QbD is used in practice to produce new pharmaceutical products at various levels, as shown in Figure 2 [9,31,32].

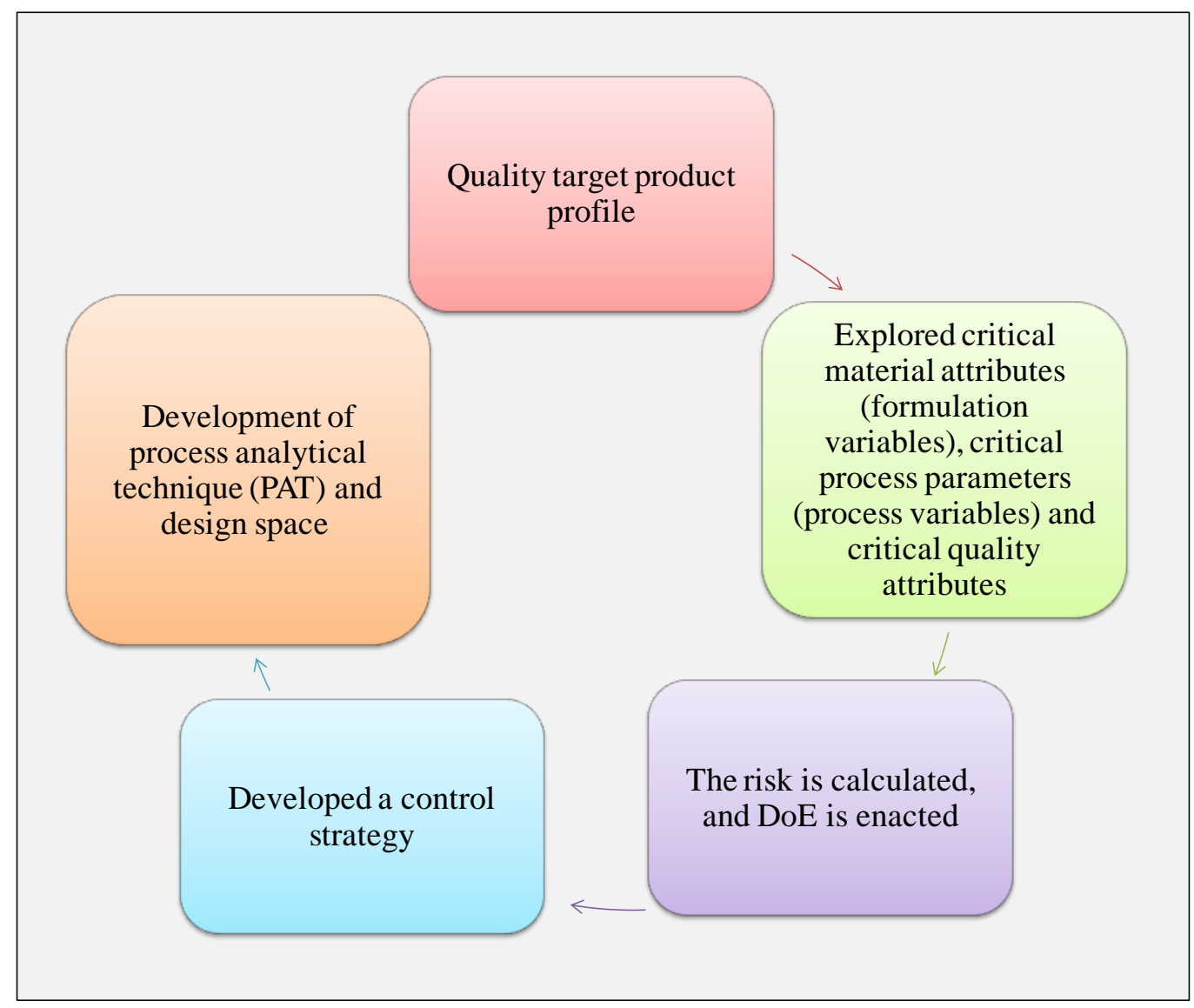

Figure 2. Steps indicating the approach of QbD.

\subsection{Design layout.}

As per ICH Q8 (R2), the design space has become a multidisciplinary framework and system of process parameters and manufacturing methods that have been shown to produce high-quality products. The developer recommends a design-based layout that is subjected to legal inspection and that is typically system and equipment-based; however, the design space chosen for the lab scale would not be applicable to commercially viable procedures. On a large level, design-space checking is therefore needed before it is established that the design space is self-contained [9]. The procedure for developing a design space for QbD elements includes providing input materials and processing of variables leading to the development of the product, followed by assessment and certification by regulatory bodies.

\subsection{Elucidation of control strategy and continuous development.}

Adjustments in the design space's layout, as in the QbD model, would not necessitate review or clearance. As a result, process improvements in terms of quality and performance can occur earlier in the product cycle, with fewer demands for post-approval. In relation to regulatory continuity, a better understanding of the manufacturing environment would aid in more efficient risk management in accordance with ICH Q9 criteria for the impact of perceived changes and production deviations on quality products [9]. 


\section{Tools of QbD}

For comprehending the aspects of $\mathrm{QbD}$ in the industrial paradigm, the implementation of successful QbD methods is essential, which include process analytical technology (PAT), risk evaluation, and design of experimentation (DoE) [9].

\subsection{Risk assessment.}

Risk evaluation is a method of organizing data to aid in risk decision-making in a risk mitigation environment. It entails detecting hazards as well as assessing and deciding exposure risks. This will be the first part of the quality-based risk management phase, followed by risk reduction and assessment stages. Risk management entails executing results to reduce or eliminate the risk and includes three components: risk identification, interpretation, and assessment. The risk management results should be reviewed in the final process to ensure that the basic tools and awareness are considered [33]. Historical facts, theoretical models, rational claims, and strategic choices are all used in the identification process to identify possible causes of hazards which led to the risk issue or challenge classification. Risk analysis needs to calculate risks based on the identified hazard; risk evaluation entails comparing the calculated risk to risk factors available through a qualitative or quantitative approach to determine the risk's significance.

ICH Q9 lists the following nine common risk management tools: (1) An evaluation of the basic risks (2) evaluation of the tree diagram (Ishikawa fishbone template, scatter diagrams, assess sheets); (3) facilitation strategies (4) initial risk analysis; (5) vulnerability scans with control laws; (6) an evaluation of malfunction and consequences; (7) review of failure, effects, and dissipation; (8) assessment of serviceability hazards; (9) statistical methods to assist. As part of the QbD implementation, risk assessment is prioritized through DoE. The techniques like Ishikawa fishbone and analysis of consequences/losses are widely used risk control approaches, either utilized separately or in combination [34-36].

\subsection{Design of experimentation (DoE).}

Initially, a risk-based assessment will be performed in addition to developing a research methodology. Testing design is an analytical, structured process to determine the relationship between process-related variables and DoE. DOE is a computational method being used to design and implement research and interpret the information generated from the experimental work. It is a type of computational modeling used to perform statistical analysis of a model, method, and material that controlled input parameters to analyze its influence on the calculated response variable. This is a wonderful tool that allows researchers to inspect variables according to a predetermined design. DoE is a practical technique for establishing relationships among process inputs and outputs in order to acquire a deeper knowledge of products and processes. It could assist in determining the ideal setting, CPPs, CMAs, and design space. This is recommended to build a design space by DoE for nonparametric exploration [31]. DoE was shown to be effective in developing different pharmacological therapies and operational conditions, and it can be used more extensively in the future years to ensure strong research performance with improved results. Various advantages of DOE are represented in Figure 3, and the classification of DOE techniques is illustrated in Figure 4. 


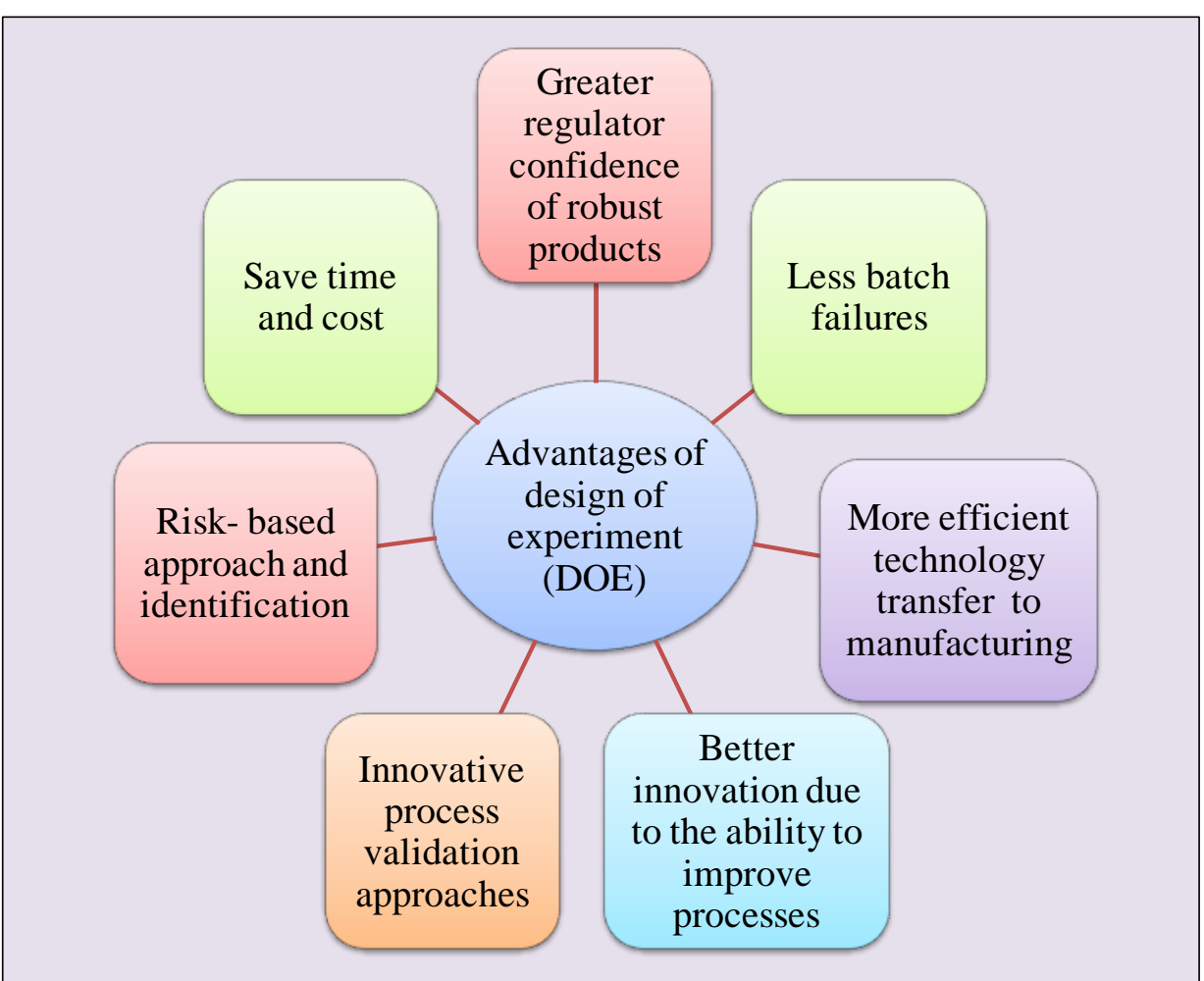

Figure 3. Advantages of design of experimentation techniques in pharmaceutical product optimization.

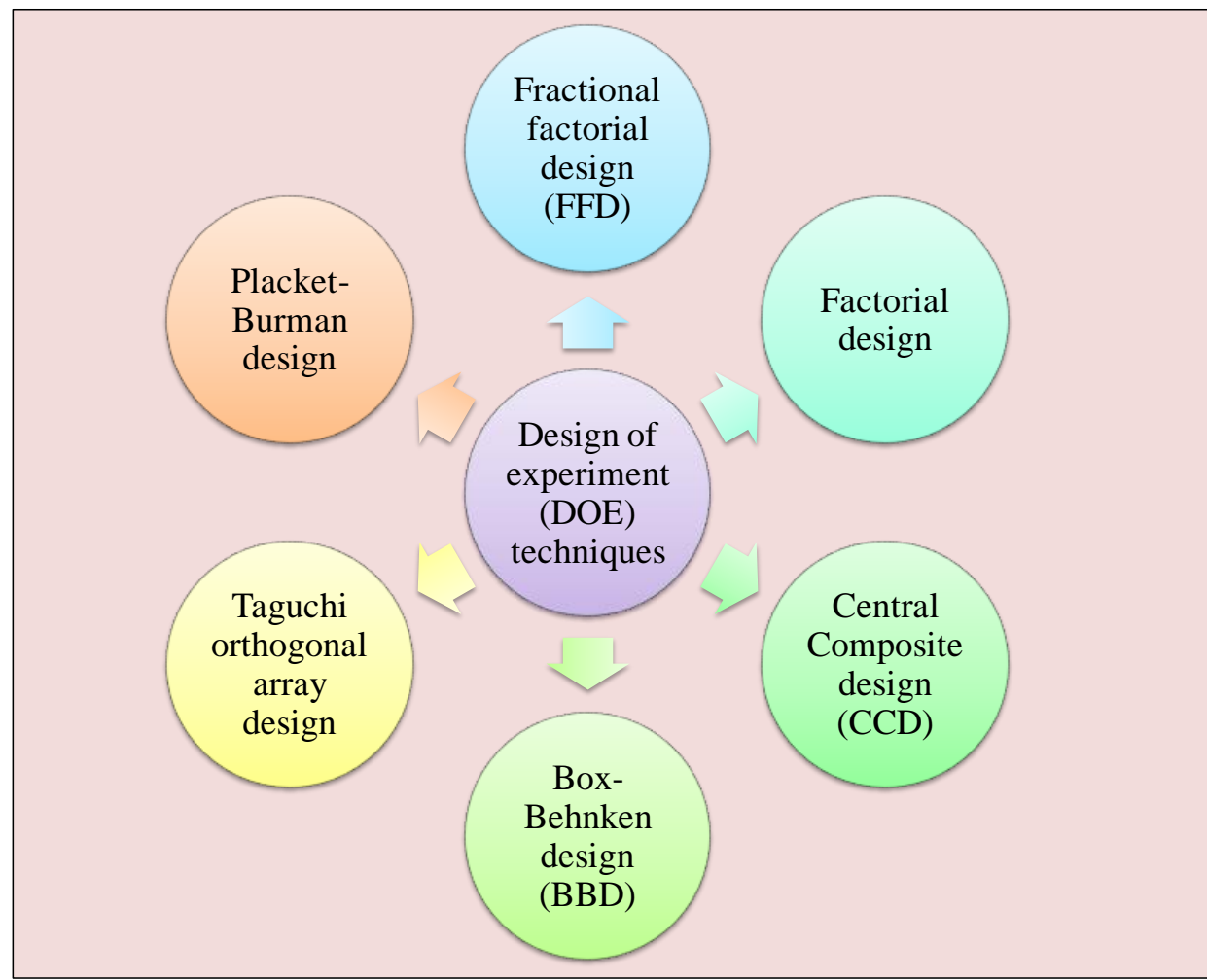

Figure 4. Classification of design of experiments techniques.

\subsection{Process analytical technology (PAT).}

PAT is described as a technique that uses actual variables throughout production to provide information to support optimized computing in order to produce a final product continuously in line with established quality and performance criteria [23]. ICHQ8 acknowledges the use of PAT to ensure cycle consistency in a design space that has already 
been developed [31]. A system is considered developed from a PAT perspective when all important causes of variability are defined and described; the system handles variance; product equity's performance can be calculated effectively and accurately [34,37].

There is a three-step method of optimization of pharmaceutical preparations and production processes, which includes monitoring, study, and design, which is often used to compile literary works. In the monitoring stage, a performance measurement program allows real-time monitoring of all CPPs and CQAs by directly or indirectly systematic techniques and suitable analysis processes to investigate the identified quality attribute, required product attributes, and processing methods. During the design stage, experiments are carried out to determine which key variables are connected to a material's role, and the process conditions and input material properties may have a major effect on finished product quality $[38,39]$. This data is being used to identify the CQA, CPP, and QTPP requirements for establishing a PATbased process control system [40].

As per FDA's PAT policy statement, process analysis could be characterized into three types: at-line, in-line, and online [13]. During at-line estimation, the sample is taken, extracted, and analyzed near the processing stream. Through online prediction, the specimens were removed from the apparatus and could be reintroduced into the flowing fluid. In the course of in-line estimation, the sample remains inside the processing stream and could be disruptive or intrusive. It is evident that PAT aids in the successful enforcement of QbD. It could perform real-time system monitoring without disruptions by bringing technological and systemic variables across online. PAT raises technology awareness which results in better quality control and register simplicity.

\section{Applications of QbD in Optimization of Microparticles and Nanotechnology-Based Drug Delivery Systems}

Nanomedicine has grown in popularity in recent decades, and research into the advancement of nanomaterials has been at the forefront of drug development. So many studies presented here indicated that microparticles, dendrimers, microemulsions, nanoemulsions, and micelles are successful drug delivery potential substitute's pharmaceutical formulations. The key benefits of these nanomaterials include increased bioavailability and sustained drug delivery, resulting in improved clinical outcomes and fewer adverse effects [41,42]. Controlling the efficiency and protection of micro- and nanomaterials is also critical. Furthermore, the large-scale transition of production processes is a barrier to industrial microand nanosystem development and clinical use. As a result, applying the QbD framework to develop nanomaterials is a strategic feature in deciding important production conditions and controlling variables that interact with the safety and quality of the finished products [43-45].

Many researchers have used this approach to evaluate CPPs, CQAs, design processes, CMAs, and quantitative strategies [46,47]. The present review discusses the most important studies that have reported the implementation of the QbD method to optimize nano-and microparticles-based systems, which commence the consistency and effectiveness of pharmaceutical products and allow for effective formulation optimization [48-51]. Raw material properties, product development, and manufacturing methods are examples of factors that require monitoring [52-54]. The specific QbD phases in the creation of micro-and nanobased systems are depicted in Figure 5. The QbD method relies on the prior recognition of critical research parameters. 


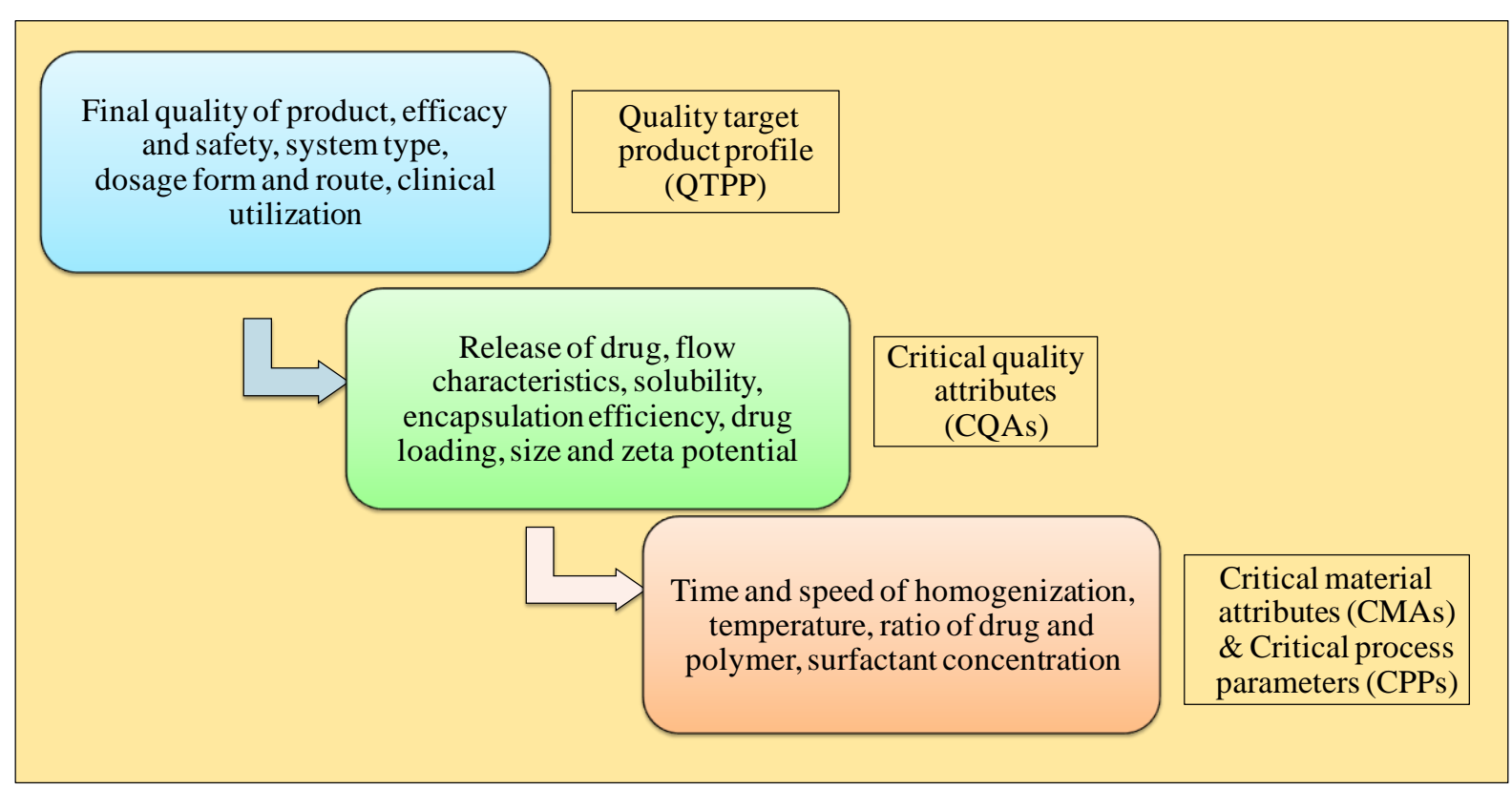

Figure 5. QBD approach steps in the micro and nanosystem implementation.

In the production of microparticles and nanotechnology-based drug delivery systems nanoparticle compositions, $\mathrm{QbD}$ has been used to promote effective drug release profile and targeting along with augmentation of pharmacodynamics, pharmacokinetics, and toxicity characteristics. DoE improves the consistency of the finished product by lowering the number of necessary experiments, which have been tested using various statistical formulas. There are several DoE design models that could help optimize the formulations of micro-and nanotechnology systems. Such examples of several researchers that utilized the approach of QbD in the optimization of micro-and nanoformulation are listed in Table 2.

Table 2. QbD for optimization of microparticles and nanotechnology-based drug delivery systems.

\begin{tabular}{|c|c|c|c|c|}
\hline Formulation & QbD approach & Dependent variables & Independent variables & Ref. \\
\hline $\begin{array}{l}\text { Dexamethasone- } \\
\text { polymeric nanoparticle }\end{array}$ & $\begin{array}{l}\text { Multiple-level full } \\
\text { factorial design }\end{array}$ & $\begin{array}{l}\text { Zeta potential, particle } \\
\text { size, \%EE, PDI }\end{array}$ & $\begin{array}{l}\text { Drug Concentration, } \\
\text { Polymer concentration, } \\
\text { Surfactant concentration }\end{array}$ & {$[55]$} \\
\hline $\begin{array}{l}\text { Curcumin loaded } \\
\text { Polymeric } \\
\text { Microparticulate }\end{array}$ & $\mathrm{CCD}$ & $\begin{array}{l}\text { Particle size, drug } \\
\text { loading, } \mathrm{PDI}, \% \mathrm{EE}, \\
\text { yield, drug release in } 2, \\
4,6,12 \text {, and } 24 \mathrm{~h}\end{array}$ & $\begin{array}{l}\text { Concentration of drug, } \\
\text { Concentration of polymer, and } \\
\text { Concentration of the polymer } \\
\text { mixture (Eudragit FS and } \\
\text { polycaprolactone) }\end{array}$ & [56] \\
\hline $\begin{array}{l}\text { Satranidazole based } \\
\text { nanoparticle }\end{array}$ & $\begin{array}{l}2^{3} \text { full factorial } \\
\text { design }\end{array}$ & $\begin{array}{l}\text { Particle size, \%EE, zeta } \\
\text { potential, dissolution } \\
\text { efficiency }\end{array}$ & $\begin{array}{l}\text { PVA concentration, } \\
\text { Aqueous phase volume, } \\
\text { Polymer concentration }\end{array}$ & [57] \\
\hline $\begin{array}{l}\text { Exemestane loaded } \\
\text { vitamin E TPGS- } \\
\text { nanoparticle }\end{array}$ & $2^{3}$ factorial design & Particle size,$\% \mathrm{EE}$ & $\begin{array}{l}\text { Polymer concentration, } \\
\text { Stirring speed, } \\
\text { TPGS volume }\end{array}$ & [58] \\
\hline $\begin{array}{l}\text { Cyclosporine A- } \\
\text { encapsulated } \\
\text { nanoparticle }\end{array}$ & $\begin{array}{l}\text { Plackett-Burman } \\
\text { design }\end{array}$ & $\begin{array}{l}\text { Zeta potential, particle } \\
\text { size, dissolution } \\
\text { efficiency, \%EE, burst } \\
\text { effect }\end{array}$ & $\begin{array}{l}\text { Concentration of drug, } \\
\text { Concentration of polymer, } \\
\text { Concentration of emulsifier, } \\
\text { Stirring speed, Solvent ratio, } \\
\text { Organic to the aqueous phase }\end{array}$ & [59] \\
\hline $\begin{array}{l}\text { Docetaxel-encapsulated } \\
\text { polyhydroxybutyrateco- } \\
\text { hydroxyvalerate } \\
\text { nanoparticle }\end{array}$ & $\begin{array}{l}\text { Plackett-Burman } \\
\text { design for } \\
\text { screening and } \\
\text { BBD main design }\end{array}$ & $\begin{array}{l}\text { Particle size, \%EE, zeta } \\
\text { potential }\end{array}$ & $\begin{array}{l}\text { Homogenizer time, } \\
\text { Polymer concentration, } \\
\text { Homogenizer speed, } \\
\text { Surfactant concentration, } \\
\text { Ultra-sonication time }\end{array}$ & [60] \\
\hline
\end{tabular}




\begin{tabular}{|c|c|c|c|c|}
\hline Formulation & QbD approach & Dependent variables & Independent variables & Ref. \\
\hline $\begin{array}{l}\text { Paclitaxel loaded } \\
\text { nanoparticle }\end{array}$ & $\begin{array}{l}\text { Plackett-Burman } \\
\text { design, BBD }\end{array}$ & $\begin{array}{l}\text { Zeta potential, \%EE, } \\
\text { particle size }\end{array}$ & $\begin{array}{l}\text { Drug concentration, } \\
\text { PLGA concentration, } \\
\text { PLGA molecular weight, } \\
\text { PLGA terminal group type, } \\
\text { Surfactant type, } \\
\text { Surfactant concentration, } \\
\text { Homogenization rate, } \\
\text { Homogenization time }\end{array}$ & {$[61]$} \\
\hline $\begin{array}{l}\text { Cetuximab loaded } \\
\text { nanoparticle }\end{array}$ & $\begin{array}{l}3^{3} \text { full factorial } \\
\text { design }\end{array}$ & $\begin{array}{l}\text { Particle size, \%EE, zeta } \\
\text { potential }\end{array}$ & $\begin{array}{l}\text { Polymer concentration, } \\
\text { Cross-linker concentration, } \\
\text { Temperature }\end{array}$ & [62] \\
\hline $\begin{array}{l}\text { Quercetin-loaded } \\
\text { nanoparticle }\end{array}$ & $\begin{array}{l}\text { Fractional factorial } \\
\text { design }\end{array}$ & $\begin{array}{l}\text { Particle size, zeta } \\
\text { potential, \%EE, PDI }\end{array}$ & $\begin{array}{l}\text { Polymer concentration, } \\
\text { Stabilizer concentration } \\
\text { Stirring speed, } \\
\text { Volumes of acetone: aqueous } \\
\text { phase }\end{array}$ & [63] \\
\hline $\begin{array}{l}\text { Loratadine dry } \\
\text { nanoparticles and } \\
\text { nanosuspension }\end{array}$ & CCD & $\begin{array}{l}\text { Particle size, } \\
\text { polydispersity index, } \\
\text { solubility, dissolution }\end{array}$ & $\begin{array}{l}\text { Drug amount, } \\
\text { Solvent to anti-solvent ratio, } \\
\text { Stabilizer type, } \\
\text { Stabilizer concentration, } \\
\text { Sonication time, Sonication power }\end{array}$ & [64] \\
\hline $\begin{array}{l}\text { Sorafenib encapsulated } \\
\text { nanoparticle }\end{array}$ & $\mathrm{BBD}$ & $\begin{array}{l}\text { Size, Dissolution at } \\
5 \mathrm{~min}, 60 \mathrm{~min}, 180 \mathrm{~min} \\
\text { and max concentration } \\
\text { of the drug, area under } \\
\text { curve drug } \\
\text { concentration }\end{array}$ & $\begin{array}{l}\text { Concentration of HPMC, } \\
\text { PVP concentration, } \\
\text { Poloxamer concentration }\end{array}$ & {$[65]$} \\
\hline $\begin{array}{l}\text { Vildagliptin } \\
\text { encapsulated Eudragit }{ }^{\circ} \\
\text { microspheres }\end{array}$ & $\begin{array}{l}\text { Plackett-Burman } \\
\text { design }\end{array}$ & $\begin{array}{l}\% \mathrm{EE} \text { and Dissolution } \\
\text { rate }\end{array}$ & $\begin{array}{l}\text { Eudragit RS- } 100 \text { concentration, } \\
\text { Span- } 80 \text { amount } \\
\text { Volume of methanol, } \\
\text { Volume of acetone, } \\
\text { Stirring speed }\end{array}$ & [66] \\
\hline $\begin{array}{l}\text { Albendazole loaded } \\
\text { chitosan microparticles }\end{array}$ & $\begin{array}{l}\text { Plackett-Burman } \\
\text { design }\end{array}$ & $\begin{array}{l}\mathrm{pH}, \text { yield, morphology, } \\
\text { dissolution rate size, } \\
\% \mathrm{EE}\end{array}$ & $\begin{array}{l}\text { Stirring time, Stirring rate, } \\
\text { Temperature, } \\
\text { pH of an ionic solution, } \\
\text { pH of chitosan solution, } \\
\text { Chitosan concentration }\end{array}$ & [67] \\
\hline $\begin{array}{l}\text { Cefadroxil encapsulated } \\
\text { Chitosan nanoparticle }\end{array}$ & $\begin{array}{l}2^{2} \text { full factorial } \\
\text { design }\end{array}$ & $\begin{array}{l}\text { PDI, zeta potential, } \\
\% \text { EE, Size, }\end{array}$ & $\begin{array}{l}\text { Polymer weight } \\
\text { Polymer concentration }\end{array}$ & [68] \\
\hline $\begin{array}{l}\text { Pioglitazone } \\
\text { Hydrochloride loaded } \\
\text { polymeric nanoparticle }\end{array}$ & $3^{2}$ factorial design & $\begin{array}{l}\text { Size, PDI, \%EE, } \\
\text { zeta potential }\end{array}$ & $\begin{array}{l}\text { Stirring rate, Stirring time, } \\
\text { Polymer concentration, } \\
\text { Surfactant concentration, } \\
\text { Amount of drug }\end{array}$ & [69] \\
\hline $\begin{array}{l}\text { Prednisolone loaded } \\
\text { polymeric nanoparticle }\end{array}$ & $2^{3}$ factorial design & $\begin{array}{l}\text { Size, drug loading, Zeta } \\
\text { potential }\end{array}$ & $\begin{array}{l}\text { Drug to polymer ratio, } \\
\text { Sonication time, } \\
\text { PLGA concentration }\end{array}$ & {$[70]$} \\
\hline $\begin{array}{l}\text { Quercetin loaded } \\
\text { polymeric nanoparticle }\end{array}$ & $\begin{array}{l}3^{2} \text { full factorial } \\
\text { design }\end{array}$ & $\begin{array}{l}\text { Size, drug release, } \\
\text { encapsulation efficiency }\end{array}$ & $\begin{array}{l}\text { Polymer concentration, } \\
\text { Pluronic F-127 amount }\end{array}$ & [71] \\
\hline $\begin{array}{l}\text { Enoxaparin sodium } \\
\text { encapsulated polymeric } \\
\text { microspheres }\end{array}$ & $\mathrm{CCD}$ & $\begin{array}{l}\% \mathrm{EE}, \\
\text { Particle size }\end{array}$ & $\begin{array}{l}\text { Eudragit FS-30D: Eudragit RS-PO, } \\
\text { Polymer concentration } \\
\text { Sodium chloride concentration }\end{array}$ & [72] \\
\hline $\begin{array}{l}\text { Quetiapine fumarate } \\
\text { loaded mucoadhesive } \\
\text { microspheres }\end{array}$ & $\begin{array}{l}2^{5-2} \text { fractional } \\
\text { factorial design }\end{array}$ & $\begin{array}{l}\text { Particle size, drug } \\
\text { release, percentage } \\
\text { mucoadhesion } \\
\text { behaviour }\end{array}$ & $\begin{array}{l}\text { Ethyl cellulose concentration, } \\
\text { Chitosan concentration, } \\
\text { Stirring speed, Type of HPMC, } \\
\text { HPMC concentration }\end{array}$ & [73] \\
\hline $\begin{array}{l}\text { Catechin hydrate } \\
\text { polymeric nanoparticle }\end{array}$ & CCD & Zeta potential PDI, size & $\begin{array}{l}\text { PLGA composition, } \\
\text { PVA composition, }\end{array}$ & [74] \\
\hline
\end{tabular}




\begin{tabular}{|c|c|c|c|c|}
\hline Formulation & QbD approach & Dependent variables & Independent variables & Ref. \\
\hline & & & $\begin{array}{l}\text { Temperature, } \\
\text { Sonication time }\end{array}$ & \\
\hline $\begin{array}{l}\text { Piroxicam polymeric } \\
\text { nanoparticle }\end{array}$ & $\mathrm{CCD}$ & $\begin{array}{l}\text { Particle size, } \\
\text { polydispersity index }\end{array}$ & $\begin{array}{l}\text { The concentration of starch and } \\
\text { drug }\end{array}$ & [75] \\
\hline $\begin{array}{l}\text { Zoledronic acid } \\
\text { polymeric nanoparticle }\end{array}$ & CCD & $\begin{array}{l}\text { Size PDI, \%EE zeta } \\
\text { potential }\end{array}$ & $\begin{array}{l}\text { Zoledronic acid content, } \\
\text { PLGA/Pluronic F68 ratio, } \\
\text { Organic to aqueous phase ratio }\end{array}$ & [76] \\
\hline $\begin{array}{l}\text { Eflornithine } \\
\text { hydrochloride solid lipid } \\
\text { nanoparticle }\end{array}$ & CCD & $\begin{array}{l}\text { Entrapment efficiency, } \\
\text { drug loading, mean } \\
\text { diameter, } \\
\text { process yield }\end{array}$ & $\begin{array}{l}\text { Drug: lipid, } \\
\text { Surfactant concentration, } \\
\text { Stirring time }\end{array}$ & [77] \\
\hline $\begin{array}{l}\text { Pentazocine } \\
\text { microspheres }\end{array}$ & $\begin{array}{l}\text { Three factors, } \\
\text { three levels Box } \\
\text { Behnken design }\end{array}$ & $\begin{array}{l}\text { Size, dissolution rate, } \\
\% \mathrm{EE}\end{array}$ & $\begin{array}{l}\text { Polymer concentration, } \\
\text { Stirring speed, } \\
\text { Surfactant concentration }\end{array}$ & [78] \\
\hline $\begin{array}{l}\text { Metformin } \\
\text { hydrochloride } \\
\text { microparticles }\end{array}$ & BBD & $\begin{array}{l}\text { Encapsulation } \\
\text { efficiency, particle size, } \\
\text { zeta potential }\end{array}$ & $\begin{array}{l}\mathrm{pH} \text { of the aqueous phase, } \\
\text { Metformin in the inner aqueous } \\
\text { phase, } \\
\text { Amount of polyvinyl alcohol, } \\
\text { Aqueous phase, Stirring rate }\end{array}$ & [79] \\
\hline $\begin{array}{l}\text { Theophylline } \\
\text { microspheres }\end{array}$ & $\begin{array}{l}2 \text { factor, } 2 \text { Level } \\
\text { CCD }\end{array}$ & $\begin{array}{l}\text { Size, \%EE, Quantity of } \\
\text { drug released }\end{array}$ & $\begin{array}{l}\text { Starch: alginate } \\
\text { Polymer: drug }\end{array}$ & {$[80]$} \\
\hline $\begin{array}{l}\text { Benzylisothiocynate } \\
\text { nanoparticles }\end{array}$ & $\mathrm{CCD}$ & $\begin{array}{l}\text { Particle size, } \\
\text { entrapment efficiency, } \\
\text { drug release }\end{array}$ & $\begin{array}{l}\text { Amount of polymer } \\
\text { Concentration of surfactant }\end{array}$ & [81] \\
\hline $\begin{array}{l}\text { Epichlorohydrin } \\
\text { microsphers }\end{array}$ & $\begin{array}{l}\text { Two-level full } \\
\text { factorial design }\end{array}$ & $\begin{array}{l}\text { Cross-linking degree } \\
\text { and drug release rate }\end{array}$ & $\begin{array}{l}\text { Concentration of epichlorohydrin } \\
\text { Duration of cross-linking }\end{array}$ & [82] \\
\hline $\begin{array}{l}\text { Nitazoxanide } \\
\text { microbeads }\end{array}$ & $\mathrm{CCD}$ & Size, $\%$ yield, and $\%$ EE. & $\begin{array}{l}\text { Percentage of chitosan, } \\
\text { Percentage of sodium } \\
\text { tripolyphosphate }\end{array}$ & [83] \\
\hline Acyclovir emulgel & CCD & $\begin{array}{l}\% \text { Yield, size, } \% \mathrm{EE}, \% \\
\text { drug loading efficiency, } \\
\text { drug release at } 0.25 \& \\
6 \mathrm{~h}\end{array}$ & $\begin{array}{l}\text { Homogenization speed, } \\
\text { Drug/polymer ratio, } \\
\text { PVA concentration }\end{array}$ & [84] \\
\hline Ansamycin nanoparticles & CCD & $\begin{array}{l}\text { \%EE, Zeta potential, } \\
\text { Size }\end{array}$ & $\begin{array}{l}\text { The concentration of polymer, } \\
\text { Concentration of surfactant } \\
\text { The ratio of organic to aqueous } \\
\text { phase }\end{array}$ & [85] \\
\hline $\begin{array}{l}\text { Clonazepam nanoparticl } \\
\mathrm{e}\end{array}$ & $\begin{array}{l}3^{2} \text { full factorial } \\
\text { design }\end{array}$ & $\begin{array}{l}\% \mathrm{EE}, \text { size, } \% \text { drug } \\
\text { release }\end{array}$ & $\begin{array}{l}\text { PLGA amount, } \\
\text { Poloxamer } 188 \text { concentration }\end{array}$ & [86] \\
\hline $\begin{array}{l}\text { Clarithromycin } \\
\text { nanoparticles }\end{array}$ & $\begin{array}{l}3^{2} \text { full factorial } \\
\text { design }\end{array}$ & $\begin{array}{l}\text { \% Drug loading, size, } \\
\% \mathrm{EE}\end{array}$ & $\begin{array}{l}\text { Time of sonication } \\
\text { Lipid amount }\end{array}$ & [87] \\
\hline $\begin{array}{l}\text { Vitamin B12 } \\
\text { orodispersible films }\end{array}$ & BBD & $\begin{array}{l}\text { Disintegration time, } \\
\text { Tensile strength, } \\
\text { Permeation, } \\
\text { Dissolution rate }\end{array}$ & $\begin{array}{l}\text { Amount of: } \\
\text { Glycerine, } \\
\text { Menthol, } \\
\text { Polymer amount }\end{array}$ & {$[88]$} \\
\hline $\begin{array}{l}\text { Ketoconazole cubosome } \\
\mathrm{s}\end{array}$ & $3^{2}$ factorial design & $\begin{array}{l}\text { Size, \%EE, drug } \\
\text { loading, PDI }\end{array}$ & $\begin{array}{l}\text { Stabilizer, } \\
\text { Surfactant amount }\end{array}$ & [89] \\
\hline Efavirenz nanopaticles & $\begin{array}{l}\text { Central composite } \\
\text { rotatable design }\end{array}$ & Size, PDI, \%EE & $\begin{array}{l}\text { Solid lipid concentration, } \\
\text { Concentration of stabilizer }\end{array}$ & [90] \\
\hline $\begin{array}{l}\text { Resveratrol polymeric } \\
\text { micelles }\end{array}$ & $\mathrm{CCD}$ & $\begin{array}{l}\text { Size, skin deposition } \\
\text { and incorporation } \\
\text { efficiency }\end{array}$ & $\begin{array}{l}\text { Amount of Pluronic P123\%, } \\
\text { Amount of Resveratrol amount }\end{array}$ & [91] \\
\hline $\begin{array}{l}\text { Clindamycin } \\
\text { hydrochloride } \\
\text { microsponges loaded } \\
\text { topical gel }\end{array}$ & CCD & $\begin{array}{l}\text { Particle size }(\mathrm{D} 50 \mu \mathrm{m}), \\
\text { Span, Micro-sponge } \\
\text { drug content }\end{array}$ & $\begin{array}{l}\text { Water (ml), Amount of span, } \\
\text { Acetone volume, Amount of } \\
\text { ethylcellulose, Amount of drug } \\
\text { and dichloromethane }\end{array}$ & [92] \\
\hline Tazarotene naoparticles & BBD & $\begin{array}{l}\text { Size, \%EE, drug } \\
\text { release, and zeta } \\
\text { potential }\end{array}$ & $\begin{array}{l}\text { Amount of surfactant, } \\
\text { Amount of lipid }\end{array}$ & [93] \\
\hline
\end{tabular}




\begin{tabular}{|c|c|c|c|c|}
\hline Formulation & QbD approach & Dependent variables & Independent variables & Ref. \\
\hline $\begin{array}{l}\text { Resveratrol } \\
\text { nanoparticles }\end{array}$ & CCD & $\begin{array}{l}\text { Size, } \% \mathrm{EE}, \text { drug } \\
\text { release, } \\
\text { Zeta potential }\end{array}$ & Amount of Lecithin and Chitosan & [94] \\
\hline $\begin{array}{l}\text { Ibuprofen nanostructured } \\
\text { lipid carriers }\end{array}$ & $\begin{array}{l}\text { Plackett-Burman } \\
\text { design followed by } \\
\text { BBD }\end{array}$ & $\begin{array}{l}\text { PDI, size, \%EE, drug } \\
\text { loading }\end{array}$ & $\begin{array}{l}\text { The concentration of surfactant, } \\
\text { Homogenization time, } \\
\text { Concentration of lipid, } \\
\text { Homogenization speed, } \\
\text { Ultra-sonication time, } \\
\text { The ratio of surfactant, } \\
\text { Ultra-sonication frequency }\end{array}$ & [95] \\
\hline L-arginine nanoparticles & $\begin{array}{l}2^{3} \text { full factorial } \\
\text { design }\end{array}$ & $\begin{array}{l}\text { Size, \%EE, and drug } \\
\text { loading }\end{array}$ & $\begin{array}{l}\text { Oleic acid concentration, } \\
\text { Poloxamer } 188 \text { concentration, } \\
\text { Sonication rate }\end{array}$ & [96] \\
\hline $\begin{array}{l}\text { Silver Sulphadiazine } \\
\text { loaded egg oil organogel }\end{array}$ & $\begin{array}{l}\text { Taguchi } \\
\text { orthogonal array } \\
\text { design }\end{array}$ & $\begin{array}{l}\text { Release, size, viscosity, } \\
\text { phase transition } \\
\text { temperature }\end{array}$ & $\begin{array}{l}\text { Organic phase ratio, } \\
\text { Temperature, Phospholipids, } \\
\text { Egg oil, Surfactant concentration, } \\
\text { Surfactant type, Mixing time }\end{array}$ & [97] \\
\hline $\begin{array}{l}\text { Luliconazole lipid } \\
\text { crystalline nanoparticles } \\
\text { for topical delivery }\end{array}$ & $\begin{array}{l}\text { Risk Estimation } \\
\text { Matrix; } \\
\text { CCD }\end{array}$ & $\begin{array}{l}\text { Size, \%EE, PDI, } \\
\text { Zeta potential }\end{array}$ & $\begin{array}{l}\text { Lipid content (glyceryl } \\
\text { monooleate), } \\
\text { Concentration of Poloxamer 407, } \\
\text { Amount of co-surfactant (PEG } \\
200 \text { ) }\end{array}$ & [98] \\
\hline $\begin{array}{l}\text { Desoximetasone } \\
\text { niosomes }\end{array}$ & CCD & Particle size, \%EE & $\begin{array}{l}\text { Amount of drug surfactant and } \\
\text { cholesterol concentration, and } \\
\text { lipids }\end{array}$ & [99] \\
\hline $\begin{array}{l}\text { Risedronate Sodium- } \\
\text { Microspheres }\end{array}$ & $\begin{array}{l}\text { Plackett-Burman } \\
\text { design }\end{array}$ & $\begin{array}{l}\text { Size, } \% \mathrm{EE}, \\
\text { Zeta potential }\end{array}$ & $\begin{array}{l}\text { Risedronate-chitosan ratio, } \\
\text { Surfactant concentration, } \\
\text { Homogenization time, } \\
\text { Temperature, } \\
\text { Homogenization speed }\end{array}$ & [100] \\
\hline $\begin{array}{l}\text { Curcumin loaded } \\
\text { nanoemulsion }\end{array}$ & $3^{2}$ factorial design & Size, zeta potential, PDI & $\begin{array}{l}\text { Surfactant mix concentration, } \\
\text { Oil phase }\end{array}$ & [101] \\
\hline $\begin{array}{l}\text { Rivastigmine loaded } \\
\text { nanostructured lipid } \\
\text { carriers }\end{array}$ & $\begin{array}{l}\text { Optimization of: } \\
\text { Formulation } \\
\text { variables by CCD, } \\
\text { Instrumental } \\
\text { parameters by } \\
\text { BBD }\end{array}$ & $\begin{array}{l}\text { Mean particle size, } \\
\text { D50 nm, D90 nm, PDI, } \\
\text { zeta potential, \%EE }\end{array}$ & $\begin{array}{l}\text { For CCD: Precirol }{ }^{\circledR} \text { ATO 5: } \\
\text { Vitamin E ratio, Tween }{ }^{\circledR} 80 \text { : } \\
\text { Phospholipon }{ }^{\circledR} 90 \text { G concentration } \\
\text { For BBD: Emulsification speed, } \\
\text { high-pressure homogenization } \\
\text { cycle, sonication amplitude }\end{array}$ & [102] \\
\hline $\begin{array}{l}\text { Erlotinib } \\
\text { hydrochloride spray- } \\
\text { dried microparticles }\end{array}$ & BBD & $\begin{array}{l}\text { \%EE, mean particle } \\
\text { size, drug release in } 168 \\
\text { hours }\end{array}$ & $\begin{array}{l}\text { The concentration of } \\
\text { polycaprolactone, Concentration of } \\
\text { polyvinyl alcohol, Inlet } \\
\text { temperature of spray dryer }\end{array}$ & [103] \\
\hline $\begin{array}{l}\text { Metronidazole } \\
\text { nanoparticle }\end{array}$ & $\begin{array}{l}\text { Fractional factorial } \\
\text { design }\end{array}$ & Size, \%EE & $\begin{array}{l}\text { Concentration of chitosan, } \\
\text { Acetic acid concentration, } \\
\text { Concentration of tripolyphosphate }\end{array}$ & [104] \\
\hline
\end{tabular}

BBD: Box-Behnken design; CCD: Central Composite design; D50 nm: volume distribution (50\% of particles with size equal or lower to the given value of D50): D90 nm: volume distribution (90\% of particles with size equal or lower to the given value of D90); \%EE: percentage entrapment efficiency; PDI: polydispersity index; TPGS: D- $\alpha$-tocopheryl polyethylene glycol 1000 succinate.

\section{Future Prospects}

Nowadays, several firms are starting to engage with QbD principle and designing frameworks to help it, as shown by incorporating one or even more QbD components into the production, indicating a positive move into $\mathrm{QbD}$ as the future development model [105]. The primary reason for $\mathrm{QbD}$ acceptance is regulatory criteria. The medicinal company needs regulatory enforcement in order to obtain the goods approved for distribution. Even so, the QbD solution produces a high-quality service by using cost-effective techniques. By offering a design space model, QbD eliminates the commonly utilized frizzed method to system 
development. Since it is not used among drug companies, it has a bright future when regulatory authorities require it. Due to the numerous benefits and flexibility of complying with regulatory bodies, companies can voluntarily follow this principle [106,107]. QbD enables the production of reproducible goods with the necessary quality characteristics. It is a method of developing biological that is systemic in order to avoid lot errors and reduce uncertainty in product consistency. Creating new drug formulations is a difficult task that costs a lot of money. Utilizing $\mathrm{QbD}$ guarantees production performance from the start, reducing costs in producing the ideal composition. As a result, it has a lot of potential in producing high-quality pharmaceutical drug products [108]. Multivariate Data Analysis (MVDA) tools like Partial Least Squares (PLS), and Principal Component Analysis (PCA) were viewed as important optimization methods for use in addition to DoE, assisting in reducing the number of variables being used in the DoE or exploring the research observations more thoroughly. It is often used to analyse data obtained through PAT tools in order to enhance production lines continuously. The combination of MVDA and PAT to monitor and adjust the system in response to materials or process fluctuations is the ideal future norm within the pharmaceutical sector $[109,110]$.

\section{Conclusions}

The numerous advantages of $\mathrm{QbD}$ are deterrence of sampling errors and variability in research studies, the requirement of less experimentation, and improved productivity. The QbD is a cost-effective time-saving strategy that uses PAT, risk assessment, and DoE as tools to understand raw materials and process parameters better, making QbD a straightforward model for the development of pharmaceutical products for the healthcare sector. Quality by design $(\mathrm{QbD})$ has the potential to contribute to design, manufacturing, and the ability to produce highly finished goods. The microparticles and nanotechnology-based formulations need complex experimentation and an extremely time-consuming process. Therefore, applying QbD tools to develop microparticles and nanotechnology-based formulations can conclude the research processes in proficient mode. $\mathrm{QbD}$ will progressively be recognized as an integral model for the effective development of nano-and micro-formulation and application of DoE in improving design process and risk management strategy. This review article highlighted the significance of QbD methodology in developing microparticles and nanotechnology-based formulations for achieving consistent efficacy and protection in pharmaceuticals.

\section{Funding}

This research received no external funding.

\section{Acknowledgments}

The authors express gratitude to Chitkara College of Pharmacy, Chitkara University, Punjab, India, for motivational support to compile this review.

\section{Conflict of Interest}

The authors declare no conflict of interest. 


\section{References}

1. International conference on harmonization of technical requirements for registration of pharmaceuticals for Human Use, 2006, https://digitalcommons.wustl.edu/cgi/viewcontent.cgi?article=1006\&context=hrpoed.

2. Cunha, S.; Costa, C.P.; Moreira, J.N.; Lobo, J.S.; Silva, A.C. Using the quality by design (QbD) approach to optimize formulations of lipid nanoparticles and nanoemulsions: A review. Nanomed. Nanotechnol 2020, 28, 102206, https://doi.org/10.1016/j.nano.2020.102206.

3. Swain, S.; Parhi, R.; Jena, B.R.; Babu, S.M. Quality by design: concept to applications. Curr. Drug Discov. Technol. 2019, 16, 240-250, https://dx.doi.org/10.2174/1570163815666180308142016.

4. Munson, J.; Gujral, B.; Stanfield, C.F. A review of process analytical technology (PAT) in the U.S. pharmaceutical industry. Curr. Pharma. Anal. 2006, 2, 405-414, https://dx.doi.org/10.2174/157341206778699582.

5. Sangshetti, J.N.; Deshpande, M.; Arote, R.; Zaheer, Z Shinde, D.B. Quality by design approach : Regulatory need. Arab. J. Chem. 2014, 10, 1-14, https://doi.org/10.1016/j.arabjc.2014.01.025.

6. Gochhayat, G.; Alam S.; Kumar, M.; Pal, P.; Singh, R.; Saini, V. Quality by design: a new practise for production of pharmaceutical products. J. Drug Deliv. Ther. 2019, 9, 416-424, https://doi.org/10.22270/jddt.v9i1-s.2370.

7. ICH Harmonised Tripartite Guideline, Quality risk management. ICHQ9, 2005.

8. Mishra, V.; Thakur, S.; Patil, A.; Shukla, A. Quality by design (QbD) approaches in current pharmaceutical set-up. Expert Opin. Drug Deliv. 2018, 15, 737-758, https://doi.org/10.1080/17425247.2018.1504768.

9. ICH Q8 (R2), Pharmaceutical Development, Part I: Pharmaceutical Development 2009, https://www.fda.gov/regulatory-information/search-fda-guidance-documents/q8r2-pharmaceuticaldevelopment.

10. ICH Q9, Quality Risk Management 2006, https://www.fda.gov/regulatory-information/search-fda-guidancedocuments/q9-quality-risk-management.

11. ICH Q10, Pharmaceutical Quality Systems 2009, https://www.fda.gov/regulatory-information/search-fdaguidance-documents/q10-pharmaceutical-quality-system.

12. US Food and Drug Administration (FDA), Pharmaceutical Quality for the 21st Century A Risk-Based Approach Progress Report, 2007, https:/www.fda.gov/about-fda/center-drug-evaluation-and-researchcder/pharmaceutical-quality-21st-century-risk-based-approach-progress-report.

13. US Food and Drug Administration (FDA), Guidance for industry PAT-A framework for innovative pharmaceutical manufacturing and quality assurance, 2004, https://www.fda.gov/regulatoryinformation/search-fda-guidance-documents/pat-framework-innovative-pharmaceutical-developmentmanufacturing-and-quality-assurance.

14. Yu, L.X.; Raw, A.; Lionberger, R.A.; Rajagopalan, R. U.S. FDA question-based review for generic drugs: A new pharmaceutical quality assessment system, J. Generic Med. 2004, 4, 239-248, http://dx.doi.org/10.1057/palgrave.jgm.4950073.

15. Rozet, E.; Lebrun, P.; Michiels, J.F.; Sondag, P.; Scherder, T.; Boulanger, B. Analytical procedure validation and the quality by design paradigm. J. Biopharm. Stat. 2015, 25, 260-268, https://doi.org/10.1080/10543406.2014.971176.

16. Arsiccio, A.; Pisano, R. Application of the quality by design approach to the freezing step of freeze-drying: building the design space. J. Pharm. Sci. 2018, 107, 1586-1596, https://doi.org/10.1016/j.xphs.2018.02.003. 
17. Beg, S.; Hasnain, M.S.; Rahman, M.; Swain, S. Introduction to quality by design (QbD): fundamentals, principles, and applications. In: Pharmaceutical Quality by Design 2019, 1-17. Academic Press, United State, https://doi.org/10.1016/B978-0-12-815799-2.00001-0.

18. Claycamp, H.G. Perspective on quality risk management of pharmaceutical quality. Drug Inf. J. 2007, 41, 353-367, https://doi.org/10.1177/009286150704100309

19. Singh, J. International conference on harmonization of technical requirements for registration of pharmaceuticals for human use. J. Pharmacol. Pharmacother 2015, 6, 185-187, https://doi.org/10.4103/0976-500X.162004.

20. Zhong, L.; Gao, L.; Li, L.; Zang, H. Trends-process analytical technology in solid oral dosage manufacturing. Eur. J. Pharm. Biopharm. 2020, 153, 187-199, https://doi.org/10.1016/j.ejpb.2020.06.008.

21. Yu, L.X. Pharmaceutical quality by design: Product and process development, understanding, and control. Pharm. Res. 2008, 25, 781-791, http://doi.org/10.1007/s11095-007-9511-1.

22. Beg, S.; Swain, S.; Rahman, M.; Hasnain, M.S.; Imam, S.S. Application of design of experiments (DoE) in pharmaceutical product and process optimization. In: Pharmaceutical Quality by Design 2019, 43-64 Academic Press, United State, https://doi.org/10.1016/B978-0-12-815799-2.00003-4.

23. Lionberger, R.A.; Lee, S.L.; Lee, L.; Raw, A.; Lawrence, X.Y. Quality by design: concepts for ANDAs. AAPS J. 2008, 10, 268-276, https://doi.org/10.1208/s12248-008-9026-7.

24. Kenett, R.S.; Kenett, D.A. Quality by design applications in biosimilar pharmaceutical products. Accreditation Qual. Assur. 2008, 13, 681-690, http://dx.doi.org/10.1007/s00769-008-0459-6.

25. Singh, L.; Sharma, V. Quality by design (QbD) approach in pharmaceuticals: status, challenges and next steps. Drug Deliv. Lett. 2015, 5, 2-8. http://dx.doi.org/10.2174/2210303104666141112220253.

26. Politis, S.N; Colombo, P.; Colombo, G.; Rekkas, D.M. Design of experiments (DoE) in pharmaceutical development. Drug Dev. Ind. Pharm. 2017, 43, 889-901, http://dx.doi.org/10.1080/03639045.2017.1291672.

27. Taticek, R.; Liu, J. Definitions and scope of key elements of QbD. In: Jameel F., Hershenson S., Khan M., Martin-Moe S. (eds) Quality by Design for Biopharmaceutical Drug Product Development. AAPS Adv. Pharm. Sci. Ser. 2015, 18, 31-46. Springer, New York, NY, https://doi.org/10.1007/978-1-4939-2316-8_3.

28. Namjoshi, S.; Dabbaghi, M.; Roberts, M.S.; Grice, J.E.; Mohammed, Y. Quality by design: Development of the quality target product profile (QTPP) for semisolid topical products. Pharmaceutics 2020, 12, 287 , https://doi.org/10.3390/pharmaceutics12030287.

29. Hinz, D.C. Process analytical technologies in the pharmaceutical industry: the FDA's PAT initiative. Anal. Bioanal. Chem. 2006, 384, 1036-1042, https://doi.org/10.1007/s00216-005-3394-y.

30. Csóka, I.; Pallagi, E.; Paál, T.L. Extension of quality-by-design concept to the early development phase of pharmaceutical R\&D processes. Drug Discov. Today 2018, 23, 1340-1343, https://doi.org/10.1016/j.drudis.2018.03.012.

31. Yu, L.X.; Amidon, G.; Khan, M.A.; Hoag, S.W.; Polli, J.; Raju, G.K.; Woodcock, J. Understanding pharmaceutical quality by design. AAPS J. 2014, 16, 771-783, http://dx.doi.org/10.1208/s12248-014-9598-3.

32. Tomba, E.;Facco, P.;Bezzo F, Barolo, M. Latent variable modeling to assist the implementation of qualityby-design paradigms in pharmaceutical development and manufacturing: a review. Int. J. Pharm. 2013, 457, 283-297, https://dx.doi.org/10.1016/j.ijpharm.2013.08.074.

33. Rathore, A.S. Roadmap for implementation of quality by design (QbD) for biotechnology products. Trends Biotechnol. 2009, 27, 546-553, https://dx.doi.org/10.1016/j.tibtech.2009.06.006. 
34. Patwardhan, K.; Asgarzadeh, F.; Dassinger, T.; Albers, J.; Repka, M.A. A quality by design approach to understand formulation and process variability in pharmaceutical melt extrusion processes. J. Pharm. Pharmacol. 2015, 67, 673-684, https://dx.doi.org/10.1111/jphp.12370.

35. Bousses, C.; Ferey, L.; Vedrines, E.; Gaudin, K. Using an innovative combination of quality-by-design and green analytical chemistry approaches for the development of a stability indicating UHPLC method in pharmaceutical products. J Pharm Biomed Anal. 2015, 115, 114-122, http://dx.doi.org/10.1016/j.jpba.2015.07.003.

36. Corredor, C.C.; Lozano, R.; Bu, X, et al. Analytical method quality by design for an online near-infrared method to monitor blend potency and uniformity. J. Pharm. Innov. 2015, 10, 47-55, https://doi.org/10.1007/s12247-014-9205-2.

37. Rathore, A.S.; Bhambure, R.; Ghare, V. Process analytical technology (PAT) for biopharmaceutical products. Anal. Bioanal. Chem. 2010, 398, 137-154, https://doi.org/10.1007/s00216-010-3781-x.

38. Read, E.K.; Shah, R.B.; Riley, B.S.; Park, J.T.; Brorson, K.A.; Rathore, A.S. Process analytical technology (PAT) for biopharmaceutical products: Part I. concepts and applications. Biotechnol. Bioeng. 2010, 105, 7684, https://dx.doi.org/10.1002/bit.22528.

39. Read, E.K.; Shah, R.B.; Riley, B.S.; Park, J.T.; Brorson, K.A.; Rathore, A.S. Process analytical technology (PAT) for biopharmaceutical products: part II. Concepts and applications. Biotechnol. Bioeng. 2010, 105, 285-295, https://dx.doi.org/10.1002/bit.22529.

40. Orlandini, S.; Pinzauti, S.;Furlanetto, S. Application of quality by design to the development of analytical separation methods. Anal. Bioanal. Chem. 2013, 405, 443-450, https://dx.doi.org/10.1007/s00216-012-63022 .

41. Sainz, V.; Conniot, J.; Matos, A.L.; Peres, C.; Zupancic, E.; Moura, L.; Silva, L.C.; Florindo, H.F.; Gaspar, R.S. Regulatory aspects on nanomedicines. Biochem. Biophys. Res. Commun. 2015, 468, 504-510, https://dx.doi.org/0.1016/j.bbrc.2015.08.023.

42. Zhang, L.; Mao, S. Application of quality by design in the current drug development. Asian J. Pharma. Sci. 2017, 12, 1-8, https://dx.doi.org/10.1016\%2Fj.ajps.2016.07.006.

43. Martin-Moe, S.; Lim, F.J.; Wong, R.L.; Sreedhara, A.; Sundaram, J.; Sane, S.U. A new roadmap for biopharmaceutical drug product development: Integrating development, validation, and quality by design. $J$. Pharm. Sci. 2011, 100, 3031-3043, https://doi.org/10.1002/jps.22545.

44. Bastogne, T. Quality-by-design of nanopharmaceuticals - a state of the art. Nanomed. 2017, 13, 2151-2157, https://doi.org/10.1016/j.nano.2017.05.014.

45. Pramod, K.; Tahir, M.A.; Charoo, N.A.; Ansari, S.H.; Ali, J. Pharmaceutical product development: A quality by design approach. Int. J. Pharm. Investig. 2016, 6, 129-138, http://doi.org/10.4103/2230-973X.187350.

46. Verma, S.; Lan, Y.; Gokhale, R.; Burgess, D.J. Quality by design approach to understand the process of nanosuspension preparation. Int. J. Pharm. 2009, 377, 185-198, http://doi.org/10.1016/j.ijpharm.2009.05.006.

47. Li, Z.; Cho, B.R.; Melloy, B.J. Quality by design studies on multi-response pharmaceutical formulation modeling and optimization. J. Pharm. Innov. 2013, 8, 28-44. https://doi.org/10.1007/s12247-012-9145-7.

48. Silva, A.C.; Lopes, C.M.; Lobo, J.M.; Amaral, M.H. Delivery systems for biopharmaceuticals. Part II: liposomes, micelles, microemulsions and Dendrimers. Curr. Pharm. Biotechnol. 2015, 16, 955-965, https://doi.org/10.2174/1389201016666150817094637. 
49. Silva, A.C.; Lopes, C.M.; Lobo, J.M.; Amaral, M.H. Delivery systems for biopharmaceuticals. Part I: nanoparticles and microparticles. Curr. Pharm. Biotechnol. 2015, 16, 940-954, https://doi.org/10.2174/1389201016666150731112532.

50. Dhiva, S.; Ranjith, K.R.; Prajisya, P.; Sona, K.P.; Narendrakumar, G.; Prakash, P.; Emilin Renitta, R.; Samrot, A.V. Optimization of keratinase production using Pseudomonas aeruginosa SU-1 having feather as substrate. Biointerface Res. Appl. Chem. 2020, 10, 6540-6549, https://doi.org/10.33263/BRIAC105.65406549.

51. Jeevanandam, J.; San Chan, Y.; Danquah, M.K. Nano-formulations of drugs: recent developments, impact and challenges. Biochimie 2016, 128, 99-112, https://doi.org/10.1016/j.biochi.2016.07.008.

52. Singh, B.; Dahiya, M.; Saharan, V.; Ahuja, N. Optimizing drug delivery systems using systematic" design of experiments." Part II: retrospect and prospects. Crit. Rev. Ther. Drug Carrier Syst. 2005, 22, 215-294, https://dx.doi.org/10.1615/CritRevTherDrugCarrierSyst.v22.i3.10.

53. Singh, B.; Kapil, R.; Nandi, M.; Ahuja, N. Developing oral drug delivery systems using formulation by design: vital precepts, retrospect and prospects. Expert Opin. Drug Deliv. 2011, 8, 1341-1360, https://doi.org/10.1517/17425247.2011.605120.

54. Chawla, A.; Sharma, P.; Pawar, P. Eudragit S-100 coated sodium alginate microspheres of naproxen sodium: Formulation, optimization and in vitro evaluation. Acta Pharm. 2012, 62, 529-545, http://doi.org/10.2478/v10007-012-0034-X.

55. Ali, H.; Kalashnikova, I.; White, M.A, Sherman, M.; Rytting, E. Preparation, characterization, and transport of dexamethasone-loaded polymeric nanoparticles across a human placental in vitro model. Int. J. Pharm. 2013, 454, 149-157, https://doi.org/10.1016/j.ijpharm.2013.07.010.

56. Hales, D.; Tefas, L.R.;Tomuță, I.; Moldovan, C.; Gulei, D.; Munteanu, R.; Porfire, A. Development of a curcumin-loaded polymeric microparticulate oral drug delivery system for colon targeting by quality-bydesign approach. Pharmaceutics 2020, 12, 1027, https://doi.org/10.3390/pharmaceutics12111027.

57. Dhat, S.; Pund, S.; Kokare, C.; Sharma, P.; Shrivastava, B. Risk management and statistical multivariate analysis approach for design and optimization of satranidazole nanoparticles. Eur. J. Pharm. Sci. 2017, 96, 273-283, https://doi.org/10.1016/j.ejps.2016.09.035.

58. Mishra, B.; Padaliya, R.; Patel, R.R. Exemestane encapsulated vitamin E-TPGS-polymeric nanoparticles: preparation, optimization, characterization, and in vitro cytotoxicity assessment. Artif. Cells Nanomed. Biotechnol. 2017, 45, 522-534, https://doi.org/10.3109/21691401.2016.1163714.

59. Rahman, Z.; Zidan, A.S.; Habib, M.J.; Khan, M.A. Understanding the quality of protein loaded PLGA nanoparticles variability by Plackett-Burman design. Int. J. Pharm. 2010, 389, 186-194, http://doi.org/10.1016/j.ijpharm.2009.12.040.

60. Vardhan, H.; Mittal, P.; Adena, S.K.; Mishra, B. Long-circulating polyhydroxybutyrate-co-hydroxyvalerate nanoparticles for tumor targeted docetaxel delivery: Formulation, optimization and in vitro characterization. Eur. J. Pharm. Sci. 2017, 99, 85-94, https://doi.org/10.1016/j.ejps.2016.12.007.

61. Yerlikaya, F.; Ozgen, A.; Vural, I.; Guven, O.; Karaagaoglu, E.; Khan, M.A.; Capan, Y. Development and evaluation of paclitaxel nanoparticles using a quality-by-design approach. J. Pharm. Sci. 2013, 102, 37483761, https://doi.org/10.1002/jps.23686.

62. Viswanadh, M.K.; Vikas, Jha, A., Reddy Adena, S.K.; Mehata, A.K.; Priya, V.; Neogi, K.; Poddar, S.; Mahto, S.K.; Muthu, M.S. Formulation and in vivo efficacy study of cetuximab decorated targeted bioadhesive nanomedicine for non-small-cell lung cancer therapy. Nanomed. (Lond) 2020, 15, 2345-2367, http://doi.org/10.2217/nnm-2020-0167. 
63. Tefas, L.R.; Rus, L.M.; Achim, M.; Vlase, L.; Țomuță, I. Application of the quality by design concept in the development of quercetin-loaded polymeric nanoparticles. Farm 2018, 66, 798-810, https://dx.doi.org/10.31925/farmacia.2018.5.9.

64. Alshweiat, A.; Ambrus, R.; Katona, G.; Csóka, I. QbD based control strategy of loratadine nanosuspensions and dry nanoparticles stabilized by soluplus. Farm. 2019, 67, 729-735, https://dx.doi.org/10.31925/farmacia.2019.4.23.

65. Park, S.Y.; Kang, Z.; Thapa, P.; Jin, Y.S.; Park, J.W.; Lim, H.J.; Lee, J.Y.; Lee, S.W.; Seo, M.H.; Kim, M.S.; Jeong, S.H. Development of sorafenib loaded nanoparticles to improve oral bioavailability using a quality by design approach. Int. J. Pharm. 2019, 20, 229-238, https://doi.org/10.1016/j.ijpharm.2019.05.064.

66. Naik, J.; Waghulde, M. Development of vildagliptin loaded Eudragit ${ }^{\circ}$ microspheres by screening design: in vitro evaluation. Int. J. Pharm. Investig. 2018, 48, 627-637, https://doi.org/10.1007/s40005-017-0355-3.

67. Leonardi, D.; Lamas, M.C.; Olivieri, A.C. Multiresponse optimization of the properties of albendazolechitosan microparticles. J. Pharm. Biomed. Anal. 2008, 4, 802-807, https://doi.org/10.1016/j.jpba.2008.08.006.

68. Basha, M.; AbouSamra, M.M.; Awad, G.A.; Mansy, S.S. A potential antibacterial wound dressing of cefadroxil chitosan nanoparticles in situ gel: Fabrication, in vitro optimization and in vivo evaluation. Int. J. Pharm. 2018, 10, 129-140, https://doi.org/10.1016/j.ijpharm.2018.04.021.

69. Canchi, A.; Khosa, A.;Singhvi, G.; Banerjee, S.; Dubey, S. Design and characterization of polymeric nanoparticles of pioglitazone hydrochloride and study the effect of formulation variables using QbD approach. Curr. Nanomater. 2017, 2, 162-168, https://doi.org/10.2174/2405461503666180501115359.

70. Acharya, S.; Guru, B.R. Prednisolone encapsulated PLGA nanoparticles: Characterization, cytotoxicity, and anti-inflammatory activity on C6 glial cells. J. Appl. Pharm. Sci. 2020, 10, 14-21, http://dx.doi.org/10.7324/JAPS.2020.104003.

71. Kumar, V.; Verma, Priya R.P.V.; Singh, S. Development and evaluation of biodegradable polymeric nanoparticles for the effective delivery of quercetin using a quality by design approach. LWT-Food Sci. Technol. 2015, 61, 330-338, https://doi.org/10.1016/j.lwt.2014.12.020

72. Hales, D.;Vlase, L.;Porav, S.A.;Bodoki, A.;Barbu-Tudoran, L.; Achim, M.;Tomuță, I. A quality by design (QbD) study on enoxaparin sodium loaded polymeric microspheres for colon-specific delivery. Eur. J. Pharm. Sci. 2017, 100, 249-261, https://doi.org/10.1016/j.ejps.2017.01.006.

73. Komati, S.; Swain, S.; Rao, M.E.B.; Jena, B.R.; Unnam, S.; Dasi, V. QbD-based design and characterization of mucoadhesive microspheres of quetiapine fumarate with improved oral bioavailability and brain biodistribution potential. Bull. Fac. Pharm. Cairo Univ. 2018, 56, 129-145, https://doi.org/10.1016/j.bfopcu.2018.09.002.

74. Ahmad, N.; Ahmad, R.; Alrasheed, R.A.; Almatar, H.M.A.; Al-Ramadan, A.S.; Amir, M.; Sarafroz, M. Quantification and evaluations of catechin hydrate polymeric nanoparticles used in brain targeting for the treatment of epilepsy. Pharmaceutics 2020, 12, 203, https://doi.org/10.3390/pharmaceutics12030203.

75. Bhatia, M.; Rohilla, S. Formulation and optimization of quinoa starch nanoparticles: Quality by design approach for solubility enhancement of piroxicam. Saudi Pharm. J. 2020, 28, 927-935, https://doi.org/10.1016/j.jsps.2020.06.013.

76. Saka, O.M.; Öz, U.C.; Küçüktürkmen, B.; Devrim, B; Bozkır, A. Central composite design for optimization of zoledronic acid loaded PLGA nanoparticles. J. Pharm. Innov. 2020, 15, 3-14, https://doi.org/10.1007/s12247-018-9365-6. 
77. Grewal, I.K.; Singh, S.; Arora, S.; Sharma, N. Application of central composite design for development and optimization of eflornithine hydrochloride-loaded sustained release solid lipid microparticles. Biointerface Res. Appl. Chem. 2021, 112, 618-637, https://doi.org/10.33263/BRIAC121.618637.

78. Jabar, A.; Madni, A.; Bashir, S.; Tahir, N.; Usman, F.; Rahim, M.A.; Jan, N.; Shah, H.; Khan, A.; Khan, S. Statistically optimized pentazocine loaded microsphere for the sustained delivery application: Formulation and characterization. Plos One 2021, 16, e0250876, https://doi.org/10.1371/journal.pone.0250876.

79. Bouriche, S.; Cózar-Bernal, M.J.; Rezgui, F.; Álvarez, A.M.R.; González-Rodríguez, M.L. Optimization of preparation method by $\mathrm{W} / \mathrm{O} / \mathrm{W}$ emulsion for entrapping metformin hydrochloride into poly (lactic acid) microparticles using Box-Behnken design. J. Drug Deliv. Sci. Technol. 2019, 51, 419-429, https://doi.org/10.1016/j.jddst.2019.03.011.

80. Okunlola, A.; Akaeze, D.I. Formulation design and optimization of theophylline microspheres containing Akidi Beans (Vigna Unguiculate) starch as polymer using central composite design. East and Central Afr. J. Pharm. Sci. 2020, 23, 16-27.

81. Parmar, A.; Kaur, G.; Kapil, S.; Sharma, V.; Sharma, S. Central composite design-based optimization and fabrication of benzylisothiocynate-loaded PLGA nanoparticles for enhanced antimicrobial attributes. Appl. Nanosci. 2020, 10, 379-389, https://doi.org/10.1007/s13204-019-01185-0.

82. Tesfay, D.; Abrha, S.; Yilma, Z.;Woldu, G.; Molla, F. Preparation, optimization, and evaluation of epichlorohydrin cross-linked enset (Enseteventricosum (Welw.) Cheeseman) starch as drug release sustaining excipient in microsphere formulation. BioMed. Res. Int. 2020, 2147971, https://doi.org/10.1155/2020/2147971.

83. Bharti, C.; Nagaich, U.; Pandey, J.; Jain, S.; Jain, N. Development of nitazoxanide-loaded colon-targeted formulation for intestinal parasitic infections: centre composite design-based optimization and characterization. Futur J. Pharm. Sci. 2020, 6, 1-17, https://doi.org/10.1186/s43094-020-00130-1.

84. Gusai, T.; Dhavalkumar, M.; Soniwala, M.; Dudhat, K.; Vasoya, J.; Chavda, J. Formulation and optimization of microsponge-loaded emulgel to improve the transdermal application of acyclovir-a DOE based approach. Drug Deliv. Transl. Res. 2020, 1-21, https://doi.org/10.1007/s13346-020-00862-w.

85. Nair, K.G.; Velmurugan, R.; Sukumaran, S.K. Formulation and optimization of ansamycin-loaded polymeric nanoparticles using response surface methodology for bacterial meningitis. Bio NanoSci. 2020, 10, 279-291, https://doi.org/10.1007/s12668-019-00713-0.

86. Shah, P.; Sarolia, J.; Vyas, B.; Wagh, P.; Ankur, K.; Kumar, M.A. PLGA nanoparticles for nose to brain delivery of Clonazepam: Formulation, optimization by $3^{2}$ Factorial design, in vitro and in vivo evaluation. Curr. Drug Deliv. 2020, https://doi.org/10.2174/1567201817666200708115627.

87. Nair, A.B.; Shah, J.; Al-Dhubiab, B.E.; Jacob, S.; Patel, S.S.; Venugopala, K.N.; Morsy, M.A.; Gupta, S.; Attimarad, M.; Sreeharsha, N; Shinu, P. Clarithromycin solid lipid nanoparticles for topical ocular therapy: Optimization, evaluation and in vivo studies. Pharmaceutics 2021, 13, 523, https://doi.org/10.3390/pharmaceutics13040523.

88. Suryawanshi, D.; Wavhule, P.; Shinde, U.; Kamble, M.; Amin, P. Development, optimization and in vivo evaluation of cyanocobalamin loaded orodispersible films using hot-melt extrusion technology: A quality by design (QbD) approach. J. Drug Deliv. Sci. Technol. 2021, 63, 102559, https://doi.org/10.1016/j.jddst.2021.102559.

89. Rapalli, V.K.; Banerjee, S.; Khan, S.; Jha, P.N.; Gupta, G.; Dua, K.; Hasnain, M.S.; Nayak, A.K.; Dubey, S.K.; Singhvi, G. QbD-driven formulation development and evaluation of topical hydrogel containing 
ketoconazole loaded $\quad$ cubosomes. Mater. Sci. $\quad$ Eng. C. $\quad \mathbf{2 0 2 1 ,} \quad 119, \quad$ 111548, https://doi.org/10.1016/j.msec.2020.111548.

90. Gurumukhi, V.C.; Bari, S.B. Fabrication of efavirenz loaded nanoformulation using quality by design (QbD) based approach: exploring characterizations and in vivo safety. J. Drug Deliv. Sci. Technol. 2020, 56, 101545, https://doi.org/10.1016/j.jddst.2020.101545.

91. Khurana, B.; Arora, D.; Narang, R.K. QbD based exploration of resveratrol loaded polymeric micelles based carbomer gel for topical treatment of plaque psoriasis: In vitro, ex vivo and in vivo studies. J. Drug Deliv. Sci. Technol. 2020, 59, 101901, https://doi.org/10.1016/j.jddst.2020.101901.

92. Dimitrovska, I.; Olumceva, T.; Markova, E.; Kostoska, M.; Taneska, L.; Petrushevska, M.; Makrievski, V.; Todorov, J.; Shalabalija, D.; Mihailova, L.; Dimovski, R.P.; Dodov, M.G.; Crcarevska, M.S. Topical gel with ethyl cellulose based microsponges loaded with clindamycin hydrochloride for acne treatment. Cellulose 2020, 27, 7109-7126, https://doi.org/10.1007/s10570-020-03283-7.

93. Sharma, S.; Kanugo, A.; Gaikwad J. Design and development of solid lipid nanoparticles of tazatorente for the treatment of psoriasis and acne: a quality by design approach. Mater. Technol. 2020, https://doi.org/10.1080/10667857.2021.1873637.

94. Saha, M.; Saha, R.D.; Ulhosna, T.; Sharker, Md. S.; Shohag, Md. S.; Islam, M.S.; Ray, S.K.; Rahman, S.G.M.; Reza, M.H. J. Drug Deliv. Sci. Technol. 2020, 63, 102480, https://doi.org/10.1016/j.jddst.2021.102480.

95. Rathod, V. R.; Shah, D. A.; Dave, R. H. Systematic implementation of quality-by-design (QbD) to develop NSAID-loaded nanostructured lipid carriers for ocular application: preformulation screening studies and statistical hybrid-design for optimization of variables. Drug Dev. Ind. Pharm. 2020, 46, 443-455, https://doi.org/10.1080/03639045.2020.1724135.

96. Patel, D.; Patel M.; Soni, T.; Suhagia, B. Topical arigine solid lipid nanoparticles: development and characterization by QbD approach. J. Drug Deliv. Sci. Technol. 2021, 61, 102329, https://doi.org/10.1016/j.jddst.2021.102329.

97. Thakur, K.; Mahajan, A.; Sharma, G.; Singh, B.; Raza, K.; Chhibber, S.; Katare, O.P. Implementation of Quality by Design (QbD) approach in development of silver sulphadiazine loaded egg oil organogel: An improved dermatokinetic profile and therapeutic efficacy in burn wounds. Int. J. Pharm. 2020, 576, 118977, https://doi.org/10.1016/j.ijpharm.2019.118977.

98. Mahmood, A.; Rapalli, V.K.; Waghule, T.; Gorantla, S.; Singhvi, G. Luliconazole loaded lyotropic liquid crystalline nanoparticles for topical delivery: QbD driven optimization, in vitro characterization and dermatokinetic assessment, $\quad$ Chem. Phys. Lipids. 2020, 234, 105028 , https://doi.org/10.1016/j.chemphyslip.2020.105028.

99. Shah, P.; Goodyear, B.; Haq, A.; Puri, V.; Michniak-Kohn, B. Evaluations of quality by design (QbD) elements impact for developing niosomes as a promising topical drug delivery platform. Pharmaceutics 2020, 12, 246, https://doi.org/10.3390/pharmaceutics12030246.

100.Elkady, O.A.; Tadros, M.I.; El-laithy, H.M. QbD Approach for novel crosslinker-free ionotropic gelation of risedronate sodium-chitosan nebulizable microspheres: optimization and characterization. AAPS PharmSciTech. 2020, 21, 14, https://doi.org/10.1208/s12249-019-1561-2.

101. Acharya, S.; Tamane, P.; Khante, S.; Pokharkar, V. QbD based optimization of curcumin nanoemulsion: doe and cytotoxicity studies. Indian J. Pharm. Educ. Res. 2020, 54, 329-336, https://doi.org/10.5530/ijper.54.2.38. 
102.Cunha, S.; Costa, C.P.; Loureiro, J.A.; Alves, J.; Peixoto, A.F.; Forbes, B.; Sousa Lobo, J.M.; Silva, A.C. Double optimization of rivastigmine-loaded nanostructured lipid carriers (NLC) for nose-to-brain delivery using the quality by design (QbD) approach: Formulation variables and instrumental parameters. Pharmaceutics 2020, 12, 599, https://doi.org/10.3390/pharmaceutics12070599.

103.Soni, G.; Yadav, K.S.; Gupta, M.K. QbD based approach for formulation development of spray dried microparticles of erlotinib hydrochloride for sustained release. J. Drug Deliv. Sci. Technol. 2020, 57, 101684, http://doi.org/10.1016/j.jddst.2020.101684.

104.Sreeharsha, N.; Rajpoot, K.; Tekade, M.; Kalyane, D.; Nair, A.B.; Venugopala, K.N.; Tekade, R.K. Development of metronidazole loaded chitosan nanoparticles using QbD approach-a novel and potential antibacterial formulation. Pharmaceutics 2020, 12, 920, https://doi.org/10.3390/pharmaceutics12100920.

105.Ter Horst, J.P.;Turimella, S.L.;Metsers, F.; Zwiers, A. Implementation of quality by design (QbD) principles in regulatory dossiers of medicinal products in the european union (EU) between 2014 and 2019. Ther. Innov. Regul. Sci. 2021, 55, 583-590, https://doi.org/10.1007/s43441-020-00254-9.

106.Sangshetti, J.N.; Deshpande, M.; Zaheer, Z.; Shinde, D.B.; Arote, R. Quality by design approach: regulatory need. Arab. J. Chem. 2017, 10, 3412-3425, https://doi.org/10.1016/j.arabjc.2014.01.025.

107. Kalyan, S.; Parle, A. Quality by design: changing outlook of pharmaceutical development. Int. J. Pharm. Sci. Res. 2019, 10, 4100-4108, https://doi.org/10.13040/IJPSR.0975-8232.10(9).4100-08.

108.Grangeia, H.B.; Silva, C.; Simões, S.P.; Reis, M.S. Quality by design in pharmaceutical manufacturing: A systematic review of current status, challenges and future perspectives. Eur. J. Pharm. Biopharm. 2020, 147, 19-37, https://doi.org/10.1016/j.ejpb.2019.12.007.

109.Joshi, A.; Rajput, A.; Verma, P.; Srivastava, S.P.; Sharma, U.; Mishra, S. Quality by Design-A tool for pharmaceutical industry that has no near end.Int. J. Pharm. Res. 2021, 13, http://dx.doi.org/10.31838/ijpr/2021.13.03.027.

110.Minatovicz, B.; Bogner, R.; Chaudhuri, B. Use of a design of experiments (DoE) approach to optimize largescale freeze-thaw process of biologics. AAPS Pharm. Sci. Tech. 2021, 22, 1-15, http://doi.org/10.1208/s12249-021-02034-6. 